\title{
Convergence, congruence et créolisation
}

Quelques réflexions à partir de l'« agglutination nominale » dans les langues créoles françaises

Convergence, congruence and creolisation: on the case of "nominal agglutination" in French-related Creoles

\section{Georges Daniel Véronique}

\section{(2) OpenEdition}

Electronic version

URL: https://journals.openedition.org/tipa/4390

DOI: 10.4000/tipa.4390

ISSN: 2264-7082

Publisher

Laboratoire Parole et Langage

Electronic reference

Georges Daniel Véronique, "Convergence, congruence et créolisation", TIPA. Travaux interdisciplinaires sur la parole et le langage [Online], 37 | 2021, Online since 23 July 2021, connection on 20 October 2021. URL: http://journals.openedition.org/tipa/4390 ; DOI: https://doi.org/10.4000/tipa.4390

This text was automatically generated on 20 October 2021.

La revue TIPA. Travaux interdisciplinaires sur la parole et le langage est mise à disposition selon les termes de la licence Creative Commons Attribution - Pas d'Utilisation Commerciale - Pas de Modification 4.0 International. 


\section{Convergence, congruence et créolisation}

Quelques réflexions à partir de l'« agglutination nominale » dans les langues créoles françaises ${ }^{1}$

Convergence, congruence and creolisation: on the case of "nominal agglutination" in French-related Creoles

Georges Daniel Véronique

\section{Introduction}

1 La congruence et la convergence sont fréquemment invoquées pour expliquer les nombreux phénomènes translinguistiques (crosslinguistic), comme les alternances codiques, les emprunts etc., qui surgissent quand des langues sont en contact (Matras 2009). Ces processus linguistiques résultent de la coexistence de langues dans un même territoire sur la longue durée (cas d'un sprachbund, voir Johanson 1998 par exemple), et de situations d'appropriation linguistique et de bilinguisme. La convergence comme la congruence sont associées à des mécanismes qui trouvent leur origine dans les conduites d'appropriation de locuteurs plurilingues (Haspelmath 1998). Les termes de congruence et de convergence sont employés tantôt comme synonymes pour décrire des processus qui affectent les matériaux (linguistic matter) et les modes d'organisation (patterns) des langues en contact (Matras 2009 : 148), tantôt pour désigner des processus linguistiques distincts qui surviennent lors de la mise en contact de langues. Baptista (2020), qui se réfère à Corne (1999: 48 et suiv.), emploie le terme de congruence plutôt que celui de convergence dans ses travaux mais prend soin de bien différencier la congruence de la convergence. L'objet de cet article est d'interroger la signification et l'emploi des notions de convergence et de congruence et de quelques autres notions connexes en linguistique des contacts de langues et d'évaluer leurs rôles dans la créolisation. 
2 L'article se propose d'examiner les conditions qui président à la mise en œuvre de la convergence et de la congruence et de comprendre comment elles s'articulent avec d'autres fonctionnements comme le transfert et l'emprunt ou la réanalyse et la grammaticalisation, également invoqués pour expliquer le changement linguistique et la créolisation. L'article souhaite décrire quelques aspects de l'intrication des facteurs qui conduisent à l'émergence et au développement des langues créoles. Comme le rappellent fort justement Kriegel, Ludwig et Pfänder (2019) dans leur discussion des travaux de DeGraff et Aboh (2017), évoquer la convergence et la congruence entre systèmes linguistiques dans la créolisation, c'est traiter de la continuité ou de la rupture des langues créoles d'avec les langues donatrices de leurs lexiques et de leurs grammaires. En dépit des continuités observables entre les créoles français et leur langue lexificatrice par exemple (DeGraff \& Aboh 2017 ; Kriegel, Ludwig et Pfänder 2019), je retiendrai que l'émergence des langues créoles suppose une rupture avec les langues contributrices de leurs lexiques ou de leurs grammaires, ce que manifestent les cas d'hybridation (hybridism) décrits par Aboh par exemple (Aboh 2015 : 305).

Cette contribution s'ouvre sur une brève caractérisation de la créolisation en tant que processus socio-historique et linguistique de développement des langues créoles. Elle aborde ensuite la relation entre la convergence et la congruence et d'autres notions connexes dans la créolisation. Dans un troisième temps, l'exemple de l'agglutination nominale dans les créoles français ${ }^{2}$, présentée par Baker (1984) comme un cas de transfert de substrat bantou dans le développement de l'Isle de France Creole (IdFC) (c'està-dire des créoles français de Maurice, de Rodrigues et des Seychelles), est analysé et discuté. Il s'agit de comprendre la réanalyse des suites françaises Déterminant + Nom (mon père, du sel, la mort, du miel etc.) en unités lexicales nouvelles dans ces langues créoles et dans les créoles français de l'Atlantique. L'examen critique de cet exemple devrait permettre de réfléchir à l'étiologie de la créolisation. L'agglutination nominale dans les langues créoles est-elle uniquement un fait de convergence, de congruence ou d'hybridation au sens d'Aboh (2015) ? Quelles réflexions peut-on formuler sur le rôle de ces processus dans l'émergence et le développement des langues créoles?

\section{La créolisation : une appropriation linguistique par des adultes dans un cadre social esclavagiste}

4 La créolisation désigne le processus socio-historique et linguistique d'émergence et de développement des langues créoles lors de l'expansion coloniale européenne et de l'instauration de l'esclavage dans les nouvelles colonies européennes à partir du $17^{\text {ème }}$ siècle (Véronique 2013). A la suite de nombreux chercheurs dont R. Chaudenson (1992), J. Lang (2009) et E.O. Aboh et M. DeGraff (2017), je considère que les procédés d'appropriation des langues dominantes de ces colonies par des populations d'esclaves adultes, alloglottes et plurilingues, sont à l'origine des langues créoles. Dans la première partie de cet article, je voudrais développer brièvement quelques aspects de cette idée. 


\subsection{Recourir aux modèles d'acquisition des langues étrangères (LE) : quels enjeux ?}

Les chercheurs qui abordent la créolisation comme une instance d'appropriation de langues étrangères (LE) font souvent porter leurs interrogations sur la contribution des langues parlées par les esclaves dans ce processus. Pour rendre compte du rôle $d u$ transfert dans la créolisation, ils ont souvent recours au modèle générativiste de Schwartz et Sprouse (1996) (Full Access Full Transfer), ou aux modèles fonctionnalistes de la processabilité de Pienemann (1998) ou de la Basic Variety (BV) (variété de base) de Klein et Perdue $(1992,1997)$. Les modèles de Pienemann et de Klein et Perdue partagent l'idée que l'appropriation et la maîtrise d'une langue étrangère sont des processus graduels, contraints chez Pienemann par des stratégies cognitives et linguistiques de traitement des unités de la langue cible - du lemme à la proposition - et par des principes sémantiques et informationnelles d'organisation des énoncés chez Klein et Perdue. Ces deux modèles partagent également la conviction que des phases spécifiques peuvent être repérées dans le continuum de développement d'une langue étrangère en fonction de la grandeur et de la complexité des unités à traiter cognitivement pour Pienemann, et autour du développement de la finitude sémantique et syntaxique pour Klein et Perdue.

6 Dans le modèle de Schwarz et Sprouse, en rapport avec des débats propres au générativisme, il est posé qu'à l'instar des enfants qui acquièrent leur langue première (L1), les apprenants adultes de langues étrangères peuvent accèder à l'ensemble des mécanismes innés dont ils disposaient lors de l'apprentissage de la L1 pour tout apprentissage linguistique nouveau, et que de plus, ils peuvent recourir sans limites aux autres ressources linguistiques acquises ultérieurement. Le modèle de Schwarz et Sprouse, retenu entre autres par J. Lang (2009), postule que le transfert des fonctionnements de la L1 vers la langue étrangère est possible sans contrainte aucune.

7 Selon le modèle fonctionnaliste de Klein et Perdue $(1992,1997)$ au contraire, les phénomènes d'interférence et de transfert inter-linguistiques sont contraints et n'apparaissent qu'à partir d'un certain degré d'élaboration grammaticale de la variété linguistique élaborée par l'apprenant. Pour Klein et Perdue, les apprenants adultes non guidés d'une LE parcourent trois grandes étapes d'appropriation qui précèdent la mise en place de systèmes linguistiques spécifiques et autonomes. Lors de la première étape, celle de la Nominal Utterance Organization (NUO) (Organisation d'énoncés à base nominale) - des éléments lexicaux repris de la langue cible (LC) sont organisés linéairement selon des contraintes pragmatiques (focus en dernier) et sémantiques (Contrôleur / Agent en premier) pour constituer des énoncés en LE. Dans la seconde étape, l'Infinite Utterance Organization (IUO) (Organisation d'énoncés à base verbale non finie), les énoncés en LC s'organisent autour du verbe non fléchi, en fonction des mêmes contraintes pragmatiques et sémantiques que durant l'étape précédente. A ce stade, une variété de base (Basic Variety) constituée de lexèmes, voire de bribes de syntagmes de la LC, se met en place. Cette étape intermédiaire est proche de la linear phase of grammar development (phase linéaire de développement grammatical) de R. Jackendoff (1999) et rappelle la mobilisation des deux premiers layers (couches) sur les cinq postulés par Heine et Kuteva (2007) lors du développement de la grammaire dans une langue nouvelle. Au troisième stade, la Finite Utterance Organization (FUO) (Organisation d'énoncés à base verbale finie), les verbes de la variété linguistique 
émergente acquièrent la flexion morphologique et la finitude syntaxique de la LC, non sans quelques dysfonctionnements idiosyncrasiques.

Dans les perspectives fonctionnalistes, on considère que les transferts interlinguistiques n'interviennent principalement qu'après le stade de la variété de base. De plus, toutes les unités et tous les fonctionnements de la langue à acquérir ne constituent pas nécessairement des cibles pour le transfert des unités de la L1. Le transfer to somewhere principle d'Andersen (1983) qui pose que le transfert interlinguistique est favorisé par l'identification des sites de la L2 où il peut s'instancier, semble guider ce processus. Donc, selon que l'on se réfère à Schwartz et Sprouse ou à des démarches fonctionnalistes, on n'attribuera pas le même rôle à l'influence des langues des populations d'esclaves dans la créolisation.

Adhérent à l'idée qu'à l'origine de la créolisation se trouve un processus d'appropriation linguistique dans le cadre social particulier de l'esclavage, j'ai recours au modèle de la variété de base (BV) développé par Klein et Perdue $(1992,1997)$. Comme Becker et Veenstra (2003), je considère que les langues créoles émergent à partir du stade IUO de développement des interlangues ou de la variété de base qu'élaborent les esclaves à l'occasion de l'appropriation non guidée du français colonial. Les nouvelles variétés linguistiques développées par les esclaves rompent les amarres avec les langues donatrices de leur lexique, voire de certaines constructions grammaticales, pour se cristalliser (Baker 1984) en langues autonomes.

\subsection{Recourir aux modèles d'acquisition des LE : quelles conséquences?}

10 La référence aux recherches sur l'appropriation des langues étrangères par des adultes pour expliquer le processus de créolisation dans sa double dimension cognitive et linguistique suppose que soient retenues les leçons de ces travaux sur la gradualité du développement des interlangues, sur les transferts inter-linguistiques et sur la dynamique des contacts inter-linguistiques.

\subsubsection{Traitement de l'input et élagage (stripping process)}

11 Les modèles de la BV et de la processabilité postulent que lors des premières phases de l'appropriation linguistique, donc également de la créolisation, le traitement cognitif des apports linguistiques en langue étrangère (input) provoque un processus d'élagage des données linguistiques reçues. Cela rejoint les thèses de Bickerton $(1981,1988)$ et de Heine et Kuteva (2007: 168), selon lesquelles les premières étapes de l'appropriation linguistique supposent l'élimination de certains marqueurs et de certains fonctionnements syntaxiques de l'input reçu et perçu, tels l'accord en genre et en nombre, la morphologie verbale désinentielle ou la morphologie dérivationnelle. A l'étape de la variété de base (BV) et aux étapes suivantes (Klein \& Perdue 1992, 1997), une restauration-reconstruction des items linguistiques est effectuée qui affectent les couches (layers) 3 à 5 - de l'adjectif et de l'adverbe à l'accord et à la subordination - de la grammaire en cours d'élaboration, si l'on se réfère au modèle de Heine et Kuteva (2007). Ainsi, selon Bickerton $(1981,1988)$ d'une part, et Heine et Kuteva (2007) d'autre part, après l'élagage initial, les langues créoles reconstruisent des articles, des marqueurs de Temps-Aspect-Mode, des marqueurs de nombre grammatical et d'interrogation, des pronoms personnels etc. Lors du déroulement de ce processus, les 
langues créoles émergentes puisent dans les langues en contact (Véronique 2007) selon des processus de convergence (voir par exemple Myers-Scotton (2002), de congruence (Baptista 2020), de transfert et de grammaticalisation (Kriegel, Ludwig \& Pfänder 2019).

\subsubsection{Les mécanismes du transfert inter-linguistique}

Dans leur ouvrage consacré aux influences translinguistiques, Jarvis et Pavlenko (2008) proposent une vision renouvelée des influences entre langues en contact. Ils concluent à la suite d'Odlin (1989: 61), que le transfert s'exerce entre tous les sous-systèmes des langues en contact (Jarvis \& Pavlenko 2008 : 46). Dans la typologie qu'ils établissent, ces auteurs distinguent aussi bien des transferts lexicaux et conceptuels que sémantiques, syntaxiques et phonologiques, fonctionnements qui se fondent sur la similitude perçue entre sous-systèmes en contact. Au-delà de cet inventaire, Jarvis et Pavlenko (2008: 154) avancent l'idée que des changements conceptuels (conceptual change) sont susceptibles de se produire lorsque des langues sont mises en contact. Ces changements interviennent lorsqu'au moins l'une des trois conditions suivantes se réalise : a) une modification des propriétés, des scripts et des représentations mentales (mental imagery) liés à une catégorie linguistique déterminée; b) un changement de la prototypicalité de certaines unités ou de certaines propriétés constitutives de la catégorie linguistique en question; et c) des modifications dans la connaissance ou dans les représentations des propriétés d'une catégorie linguistique. Ces changements sont susceptibles de se produire quand des adultes doivent catégoriser des actions, des événements et des objets dans leur nouvelle langue. L'un des procédés auxquels ces locuteurs ont recours est alors celui de la convergence, définie par Jarvis et Pavlenko (2008: 155 et suivantes) comme la création d'un domaine conceptuel nouveau, qui incorpore des propriétés attestées dans toutes les langues en contact, et d'où résulte un fonctionnement linguistique inédit.

\subsubsection{Transfert et copiage : une question de terminologie ?}

13 En réaction à la notion de transfert qui recouvre également l'emprunt (borrowing), usitée dans les travaux sur l'acquisition (Jarvis \& Pavlenko 2008), L. Johanson (2002) a proposé un cadre d'analyse où la notion d'emprunt est remplacée par celle de copiage (copying) : soit un copiage global qui concerne un bloc de matériaux linguistiques de la langue modèle transféré dans la langue réplique, soit un copiage sélectif, qui porte sur seulement certains des constituants dudit bloc. Le copiage se réalise dans un 'basic code', un code de base qui n'est pas sans rappeler le matrix language (langue matrice) de Myers-Scotton (2002) (voir infra). L'avantage principal du modèle du code-copying de L. Johanson est de mettre en lumière le caractère créatif et inédit du copiage global ou sélectif dans la langue récipiendaire. La notion de copiage comme celle du transfert implique un point d'atterrissage dans la langue récipiendaire (cf. Andersen 1983). Au vu de la définition de la créolisation comme acquisition d'une LE par des adultes, il me semble cohérent de conserver la notion de transfert, étayée par d'importantes recherches en acquisition des langues et en matière de bilinguisme, et redéfinie par Jarvis et Pavlenko (2008), lors de l'étude du développement des langues créoles. 


\subsection{Appropriation et actes d'identité dans la créolisation}

Depuis Weinreich (1953) et Haugen (1956), l'idée s'est imposée que des contacts de langues produisent chez le locuteur locus du contact, des identifications interlinguales susceptibles d'entraîner l'apparition de diaphonèmes et de diamorphèmes, c'est-à-dire d'unités linguistiques qui enjambent les langues en contact, unités perçues par les locuteurs comme identiques et qu'ils associent de ce fait. Ces phénomènes concernent des matériaux linguistiques ou leurs modes d'organisation. La notion de changement conceptuel défendue par Jarvis et Pavlenko (2008) décrit précisément les conditions d'activation de ces effets translinguistiques.

Poser que l'appropriation de la langue dominante de la colonie par des subalternes plurilingues, est à l'origine de la créolisation, c'est accepter que la langue créole émergente obéisse à une dynamique linguistique qui résultent tout autant de transferts que d'éventuelles convergences et congruences entre langues mises en contact, et encore, d'autres procédés généraux d'apprentissage. Le processus de créolisation au niveau individuel s'associe à des processus collectifs au niveau de la communauté émergente des locuteurs. Le processus d'appropriation de la langue dominante de la colonie par des apprenants adultes plurilingues n'explique que partiellement l'apparition des langues créoles. En effet, la cristallisation des variétés d'apprenants et la rupture qu'elles marquent ainsi avec la langue dominante de la colonie ne sont possibles que parce que se développe parmi les esclaves une socialisation qui constitue la condition sine qua non de l'émergence de ces langues, et dont les phénomènes sociolinguistiques de focalisation (focusing) décrits par Lepage et Tabouret-Keller (1985) portent témoignage.

\section{Convergence, congruence et créolisation}

Dans cette seconde partie, je souhaite aborder successivement la convergence et la congruence, telles qu'elles sont définies par différents auteurs, suivant différents cheminements conceptuels, ainsi que d'autres notions annexes, avant d'en arriver à la formulation de définitions opératoires. Tout d'abord, j'analyserai brièvement le texte fondateur de Gumperz et Wilson (1971). Je signalerai également quelques autres emplois de la notion de convergence avant d'en venir à une discussion des propositions de Myers-Scotton (2002) sur la convergence et la créolisation. Je commenterai ensuite, l'article de Baptista (2020) consacré au rôle de la congruence dans la créolisation. J'aborderai enfin l'article de Kriegel, Ludwig et Pfänder (2019) qui analyse, entre autres, les notions de convergence et de congruence. Je conclurai cette partie théorique par une mise en perspective de ces notions en vue de l'analyse de l'affixation nominale dans les créoles français.

\subsection{La convergence à Kupwar selon Gumperz et Wilson (1971)}

17 Pour Matthews (2005: 75), la convergence linguistique désigne: i) l'ajustement psychosocial et linguistique entre locuteurs en interaction, et ii) le processus historique qui conduit des langues en contact à développer et à partager des identités de structure et de fonctionnement. Le locuteur étant le locus du contact des langues (Weinreich 1953), l'ajustement en interaction et le changement linguistique diachronique 
sollicitent nécessairement des mécanismes linguistiques et cognitifs dont le locuteur plurilingue est le siège. Cela est fort bien illustré par Gumperz et Wilson (1971), qui étudient la diffusion des traits linguistiques entre langues en contact à Kupwar (Inde). Dans leur article de 1971, Gumperz et Wilson montrent comment les interactions langagières entre groupes sociaux résidant à Kupwar et leurs usages alternés de langues dravidiennes (le kannada principalement) et indo-aryennes (le marathi et l'urdu) contribuent à la convergence entre ces systèmes linguistiques. La longue cohabitation linguistique attestée à Kupwar se caractérise par le fait que les langues vernaculaires indo-aryennes et dravidiennes sont usitées au sein des foyers alors que les contacts sociaux externes requièrent des réductions et des adaptations de ces mêmes variétés linguistiques. Cela conduit des langues typologiquement distinctes à présenter des fonctionnements grammaticaux analogues dans des domaines comme l'accord, l'emploi des possessifs et des démonstratifs ou encore de la copule. Selon Gumperz et Wilson (1971 : 151), la diffusion linguistique par convergence ne diffère de la pidginisation et de la créolisation, autres processus de diffusion, qu'en termes de degré.

\subsection{La convergence dans l'étude des contacts de langues}

De Silva-Corvolán (1994 : 4 et suivantes) à Matras (2009) et Sebba (2009), de nombreux auteurs œuvrant dans le domaine de la linguistique des contacts de langues ont proposé des définitions de la notion de convergence, souvent en rapport avec celle de congruence. Pour Silva-Corvolán, la convergence désigne une similitude structurale entre certains aspects grammaticaux de langues en contact sous l'action du transfert. Pour cette auteure, le contact inter-linguistique est susceptible d'accélérer des changements déjà en cours dans la langue récipiendaire. Matras (2009: 238 et suiv.) reprend la même définition de convergence et l'associe à la théorie de la grammaticalisation, et notamment à la grammaticalisation induite par les contacts inter-linguistiques. Sebba (2009) analyse, lui, l'alternance codique en tant que phénomènes de convergence et de congruence. Selon cet auteur, l'identification des congruences inter-linguistiques est à la base de l'alternance codique, laquelle contribue puissamment à des convergences locales, et dans une perspective diachronique à la convergence globale.

\subsection{Convergence et créolisation : les propositions de Myers-Scotton (2002)}

19 Myers-Scotton (2002: 164 et suivantes) décrit la convergence comme un mécanisme de changement linguistique qui engendre un rapprochement entre langues en contact. Selon Myers-Scotton, le mécanisme de la convergence est fondé sur le fractionnement de la structure lexicale abstraite d'une variété linguistique, la langue récipiendaire, et sa combinaison avec des éléments de la structure lexicale abstraite d'une autre variété linguistique avec laquelle elle est en contact, la langue donatrice. Cela conduit à une restructuration des relations grammaticales dans la langue récipiendaire et à l'intégration de morphèmes grammaticaux de surface de la langue donatrice dans cette langue. Dans le cadre de sa modélisation linguistique et cognitive des fonctionnements bilingues (la Matrix Language Frame (MLF) Model) et du classement des morphèmes qui 
l'accompagnent (4-M model) (Myers-Scotton \& Jake 2009), Myers-Scotton (2002) formule cinq hypothèses qui font de la convergence le moteur central de la créolisation.

Myers-Scotton (2002) part de l'idée que les langues parlées par les esclaves fournissent le cadre morphosyntaxique 'invisible' des langues créoles. Sa seconde hypothèse porte sur le lexique des langues en contact; elle considère que les morphèmes de contenu (content morphemes) des langues créoles émergentes proviennent principalement de la langue dominante de la colonie, la langue des colons européens. La troisième hypothèse de Myers-Scotton (2002 : 283) est ainsi formulée : des morphèmes de contenu (content morphemes) du superstrat, la langue des colons, sont susceptibles d'être reconfigurés en morphèmes de système (system morphemes) afin de satisfaire aux contraintes du cadre morphosyntaxique abstrait de la langue émergente, cadre basé sur une langue matrice (Matrix Language) composite façonnée par les langues du substrat, les langues parlées par les esclaves. Pour soutenir cet idée, Myers-Scotton emprunte à Baker (1984) l'exemple du lexème français petit qui en créole mauricien deviendrait un marqueur nominal ti-, comparable à un marqueur de classe bantou (cf. le tableau 2 infra). La quatrième hypothèse de Myers-Scotton est que les morphèmes de système précoces (early system morphemes) en provenance du superstrat peuvent être mis à contribution dans les langues créoles s'ils sont récupérés avec leurs têtes lexicales. Pour MyersScotton (2002: 280), le traitement de l'article français dans l'agglutination nominale en créole mauricien et en créole haïtien sous l'influence du système des marqueurs de classe bantous illustre cette prévision. Enfin, la dernière hypothèse de Myers-Scotton est que les morphèmes de système tardifs (late system morphemes) des langues dominantes européennes ne peuvent être empruntés tels quels par le cadre morphosyntaxique des langues créoles, d'où l'absence du «il» impersonnel de la langue dominante par exemple, ou l'emploi d'un morphème de contenu comme fr. 'après' en tant que marqueur d'aspect dans les créoles français.

\subsection{La congruence selon Baptista}

21 Baptista, Gelman, et Beck (2016) relèvent que la notion de transfert ne rend compte que des résultats des contacts inter-linguistiques effectifs alors que des similitudes de matériaux et de fonctionnement entre langues mises en contact peuvent précéder tout contact. Ces cas de convergence isomorphique sont favorables à des chevauchements et à des transferts inter-linguistiques. Cette définition n'est pas très éloignée de celle que propose P. Muysken (2000: 122 et suiv.) pour ce qu'il nomme la lexicalisation congruente (congruent lexicalization). La convergence isomorphique comme la lexicalisation congruente supposent l'existence de diamorphèmes entre langues en contact ainsi que des équivalences structurelles afin que le mélange inter-linguistique (code-mixing) puisse se produire. On retrouve là également le transfer to somewhere principle de R.W. Andersen (1983).

Baptista (2020) distingue la convergence aréale, telle qu'elle a été décrite, entre autres, par Gumperz et Wilson (1971), Johanson (2002) ou Olko, Borges et Sullivan (2018), associée à un contact des langues dans la durée, de la convergence isomorphique, où des traits présents antérieurement dans les langues mises en contact, se trouvent renforcés par la nouvelle situation de contact (voir également ce qu'en dit SilvaCorvolán 1994). Baptista oppose donc la congruence qui résulte de la convergence aréale à la congruence de fait, due à une convergence isomorphique. Selon cette 
auteure, c'est ce dernier type de convergence qui explique partiellement l'émergence des langues créoles, cependant la congruence n'est qu'un des facteurs de la créolisation (Baptista 2020 : 162). Baptista évalue la congruence entre les entités de deux langues mises en présence selon trois dimensions : la forme, la fonction ou la signification et la position syntaxique (à distinguer du rôle syntaxique) (Baptista 2020 : 169). Elle propose un modèle de recouvrement (mapping) entre matériaux linguistiques et ordonnancement des unités dans les deux langues qui présentent des convergences isomorphiques pour expliquer le développement des langues créoles (Baptista 2020 : 180 et suivantes). Pour illustrer le rôle de la congruence dans la créolisation, Baptista évoque, entre autres, l'exemple de l'agglutination nominale dans les créoles français, qui constituerait, selon elle, un cas de convergence isomorphique entre les langues bantoues et le français.

\subsection{La synthèse de Kriegel, Ludwig et Pfänder (2019)}

23 Kriegel, Ludwig et Pfänder (2019) définissent la notion de convergence à partir de Gumperz et Wilson (1971) et de Bollée (2007). Parmi les idées formulées par Bollée (2007), ces auteurs retiennent que la convergence suppose: a) l'existence d'une similitude perçue entre traits structuraux de langues en contact ; b) le constat de traits linguistiques communs dans les langues en contact et c) le renforcement d'une tendance de la langue récipiendaire par les fonctionnements de l'autre langue, qui la conforte de ce fait. La définition de la convergence retenue par Kriegel et al., est de ce fait proche de celle de convergence isomorphique défendue par Baptista, Gelman et Beck (2016).

24 Pour Kriegel et al., la convergence est le produit d'une copie couverte ou ouverte de la langue A dans la langue B. Il s'agit d'un processus dynamique qui sollicite plusieurs paramètres (actionnel, cognitif et typologico-systémique). "La convergence présuppose donc une copie, alors que - à l'inverse - une copie peut exister sans convergence » (Kriegel et al. 2019 : 325). La congruence, au contraire, est définie comme une notion typologico-structurelle et statique. Kriegel et al. relient la convergence à la grammaticalisation grâce à la notion de « tendance » formulée par Bollée.

25 "C'est pourquoi nous pensons que ce sont ces tendances visées par la définition de Bollée qui jouent un rôle essentiel dans le concept de la convergence, dans son application à l'émergence des langues créoles, et qu'il s'agit plus précisément de tendances de grammaticalisation » (Kriegel et al. $2019: 327)$

26 Le contact linguistique fonctionne comme un catalyseur, facilitant l'évolution rapide d'une construction partiellement grammaticalisée vers une grammaticalisation plus avancée. Cette convergence en acte conduit inéluctablement à une " grammaticalisation partagée » (shared grammaticalization) (Kriegel et al. $2019: 329$ ).

27 Je partage avec Kriegel et al., l'idée qu'à l'origine de la convergence se trouve des échanges inter-individuels plurilingues, et que comme toutes les activités d'appropriation, la convergence présente une face cognitive et sociale, et une face linguistique. Cependant, pour ma part, je ne différencierai pas entre les notions de congruence et de convergence, dont les usages sont finalement fluctuants mais plutôt identiques. J'admettrai que la congruence et la convergence isomorphique, ainsi que d'autres notions connexes telle la lexicalisation congruente de P. Muysken (2000) sont 
fondées sur la perception de similitudes dans les fonctionnements des langues en contact et sont donc des notions interchangeables.

\subsection{Vers une définition de la convergence et de la congruence}

Les travaux de Gumperz et Wilson (1971) et de Myers-Scotton (2002) fournissent une explication des dimensions socio-linguistiques et cognitives de la convergence. Ceux de Baptista (2020) permettent d'appréhender les mêmes dimensions par la mise en œuvre de la congruence. Pour ces chercheurs, la convergence, tout comme la congruence, sont des processus unitaires qui conduisent à de nouvelles combinaisons d'unités et de fonctionnements dans les langues en contact. Pour d'autres comme olko, Borges et Sullivan (2018) par exemple, la convergence est la résultante d'une somme de mécanismes de changement linguistique, dont la grammaticalisation, l'emprunt lexical et grammatical, la réanalyse et la perte de catégories non attestées dans la langue modèle.

Ces différentes définitions ne divergent pas tant qu'il y paraît. Occupés à définir la convergence ou la congruence, des chercheurs comme Myers-Scotton, Gumperz et Wilson ou Baptista ne se sont pas préoccupés de savoir si ces notions devaient être pensées comme des processus unitaires de réorganisation des langues en contact ou comme la résultante de plusieurs procédés distincts, isolables mais associés. À l'instar d'autres mécanismes sollicités pour expliquer la créolisation (la grammaticalisation, la réanalyse, le transfert interlinguistique et l'analogie intralinguistique par exemple), la convergence et la congruence font intervenir des dimensions acquisitionnelles (Haspelmath 1998). Pour qu'un phénomène de convergence ou de congruence se produise, les fonctionnements entre langues en contact doivent susciter une identification interlinguale chez le locuteur locus du contact de langues (Weinreich 1953), donc qu'une activité procédurale peu consciente soit déclenchée chez ce locuteur. C'est cette condition première qui explique un traitement particulier de l'input linguistique lors de la créolisation, traitement qui produit également des réanalyses, des transferts et des grammaticalisations.

La convergence et la congruence peuvent être définies comme des processus fondés sur un transfert linguistique ciblé (le transfer to somewhere principle de R.W. Andersen 1983) qui selon la dynamique des langues en contact, suscitent des réanalyses des langues modèles, c'est-à-dire des restructurations produisant de nouvelles significations grammaticales dans les langues répliques, et favorisent des grammaticalisations. Si le lien est direct entre la congruence, la convergence et la réanalyse, c'est-à-dire la réinterprétation des unités et catégories de la langue modèle, il est indirect en ce qui concerne la grammaticalisation (Hopper \& Closs-Traugott 1993 : 220). La convergence et la congruence contribuent au même titre que d'autres processus au développement de la grammaticalisation dans les langues émergentes

\section{L'agglutination nominale dans les langues créoles françaises : congruence et convergence?}

31 L'agglutination nominale, c'est-à-dire "la réunion en une seule unité de deux ou plusieurs termes ordinairement distincts » (Dubois et al. 1973:18) tels mon père, du sel, la mort, du miel etc., dans les créoles français a été longuement étudiée depuis Fournier 
et Wittman (1982) ${ }^{3}$ qui désigne sous ce terme la réanalyse des syntagmes français en entités lexicales uniques dans les créoles de l'Océan Indien ${ }^{4}$. Comme le rappelle Ladhams (2012), une intense polémique, que je ne souhaite pas aborder ici, s'est développée autour des propositions de Baker (1984). Dans cette troisième partie, je présenterai tout d'abord succinctement les propositions de Baker (1984), augmentées de celles de Henri et Bonami (2019). Je m'interrogerai ensuite, sur le bien-fondé d'expliquer l'agglutination nominale des créoles IdFC comme un cas de transfert et de convergence ou de congruence en élargissant le débat aux autres créoles français.

\subsection{L'agglutination nominale dans les créoles français.}

\subsubsection{L'analyse de Baker (1984)}

P. Baker (1984) distingue deux types d'agglutination en créole mauricien: a) l'agglutination consonantique qui frappe les lexèmes mauriciens (mau.) dérivés d'un étymon français (fr.) à initial vocalique, exemples fr. âme > mau. nam, fr. encre > mau. lank etc., (le segment consonantique provient soit d'une consonne épenthétique de liaison, soit de l'article français), et b) l'agglutination syllabique où une syllabe dérivée de l'article français (mau. di, li etc.) s'agglutine à l'étymon lexical français pour former des lexèmes créoles, exemples lera (rat), dizef (œuf), lizje (yeux) etc. P. Baker relève que ce second type d'agglutination est plus fréquent dans les créoles de l'Océan Indien, à l'exception du réunionnais, qu'en créole d'Haïti par exemple (voir tableau 1 infra).

Tableau 1

Nombre de mots agglutinés à initial syllabique en haïtien et dans quatre créoles de l'Océan Indien (adapté de Baker 1984)

\begin{tabular}{|l|l|l|l|l|l|l|}
\hline $\begin{array}{l}\text { Articles } \\
\text { français }\end{array}$ & $\begin{array}{l}\text { Items syllabiques créoles } \\
\text { agglutinés }\end{array}$ & Haïtien & Mauricien & Réunionnais & Rodriguais & Seychellois \\
\hline du, de l', des & di-, dil-/del-, diz-/dez- & 4 & 34 & 8 & 27 & 29 \\
\hline le, les & li-/liz-, lez-/le- & 10 & 62 & 1 & 46 & 45 \\
\hline la & la- & 98 & 375 & 3 & 264 & 370 \\
\hline Total & & 112 & 471 & 12 & 337 & 444 \\
\hline
\end{tabular}

Fort de ce constat, Baker (1984) postule que la présence massive des formes agglutinées dans les créoles de Maurice, de Rodrigues et des Seychelles est due à la présence d'esclaves bantouphones à Maurice durant la période de formation des créoles dits de l'Isle de France ( $I d F C)$, période qu'il situe entre 1773 et 1810 . Baker (1984:111) esquisse un scénario d'acquisition où les esclaves bantouphones, majoritaires à Maurice, réanalysent les séquences Déterminant + Nom du français en items lexicaux uniques du fait d'un transfert de leurs connaissances des marqueurs de classes bantous. Il s'agirait également d'un cas de convergence isomorphique (Baptista 2020). Une des conséquences de cette appropriation lexicale par transfert est de permettre la création 
de doublets lexicaux : fwa (fois) lefwa (foie), ver (verre) lever (ver), vwal (le voile) lavwal (la voile) etc.

\subsubsection{La reformulation de Henri et Bonami (2019)} créoles français en l'abordant sous un jour différent, celui des facteurs qui favorisent l'agglutination du déterminant en mauricien. Ils distinguent entre les lexèmes agglutinés alternants (la forme nue et la forme agglutinée sont usitées, exemple mau. dans / ladans (fr. danse) et les lexèmes agglutinés non alternants ou fixes, mau. lapli (fr. pluie).

À partir d'une analyse quantitative et statistique fondée entre autres, sur le Diksioner Morisien (Carpooran 2011), Henri et Bonami dégagent les facteurs qui favorisent l'émergence de l'agglutination nominale en mauricien; certains de ces déterminants avaient déjà été signalés par Baker (1984). Ils confirment ainsi que les étymons français monosyllabiques, à initiale vocalique, de genre grammatical féminin, de date ancienne, de fréquence d'emploi élevée et souvent accompagnés du défini singulier français la sont les meilleurs candidats à l'agglutination nominale en mauricien. Ils notent que dans la liste des mots anciens de cette langue, les étymons de genre féminin en français donnent davantage lieu à des formes agglutinées que les étymons masculins, ce qui confirmerait une influence bantoue dans les créoles IdFC car le déterminant français la est davantage susceptible de rappeler un marqueur bantou aux créateurs du créole mauricien. Henri et Bonami (2019: 25) ajoutent cependant : «(...) après la période de créolisation, l'adoption généralisée du créole comme langue maternelle par les esclaves a entraîné la disparition de l'influence bantoue, et donc la disparition de la préférence pour l'agglutination au féminin ».

\subsection{Bilan}

Comme le titre de son article l'indique, le projet de Baker (1984) est de prendre pour point de départ un phénomène lexical remarquable, la différence numérique entre les cas d'agglutination nominale dans les créoles de Maurice, de Rodrigues et des Seychelles d'une part et de la Réunion d'autre part, pour établir que les créoles IdFC présentent une évolution différente du créole réunionnais et pour dégager un scénario de créolisation des créoles $I d F C$ où le transfert de structures des $\mathrm{L} 1$ bantoues joueraient un rôle majeur. Le modèle MLF de Myers-Scotton (2002) précise cette convergence en formulant des thèses précises sur le rôle des morphèmes de contenu (content morphemes) et des morphèmes de système précoces et tardifs (early et late system morphemes) des langues en contact dans l'émergence des langues créoles. De MyersScotton (2002) à Baptista (2020), de nombreux chercheurs s'accordent à trouver que l'exemple repéré par Baker représente bien, soit un exemple de convergence (MyersScotton 2002), soit un exemple de congruence (Baptista 2020).

TIPA. Travaux interdisciplinaires sur la parole et le langage, 37 | 2021 


\section{Un réexamen de l'agglutination nominale dans les créoles français}

Sans vouloir nier l'éventuel bien-fondé de la thèse de Baker (1984) et de Henri et Bonami (2019), il paraît nécessaire d'élargir le débat afin d'être en mesure d'assigner à la convergence et à la congruence leur juste place dans l'étiologie de l'agglutination nominale dans les créoles français, et au-delà dans la créolisation. A la lumière des définitions de convergence et de congruence proposées supra, je souhaite interroger le phénomène de réanalyse lexicale qu'est l'agglutination nominale en conservant à l'esprit que tout transfert suppose le repérage de sites analogues dans la langue cible et que toute convergence isomorphique ou toute congruence implique des identités de fonctionnement perçus avant même les contacts inter-linguistiques. Je voudrais montrer dans la dernière partie de cet article que l'agglutination nominale dans les créoles français ne saurait être considérée comme un strict cas de convergence ou de congruence, voire d'hybridation (hybridism) au sens d'Aboh (2015), en dépit des affirmations de Baker (1984), et de Myers-Scotton (2002) et de Baptista (2020) à sa suite. Je commencerai par examiner sommairement la question des marqueurs de classe dans les langues bantoues et son acquisition. J'étudierai ensuite, dans la section suivante, l'emploi des formes agglutinées, variables et invariables dans les créoles français avant de conclure.

\subsection{Le système des marqueurs de classe des langues bantoues}

La forme caractéristique d'un nom dans une langue bantoue comporte une racine lexicale (Van de Velde 2019) ou base substantivale (Creissels 1991) accompagnée d'un morphème de flexion ${ }^{5}$. Ainsi, en Swahili, -tu (homme), donne $m$-tu (1 singulier + homme +) 'un homme', wa-tu (2 pluriel + homme) ('des hommes) (Van de Velde); la base substantivale est précédée de deux marqueurs de classe différents ( $m$ - et wa). En tswana, (Creissels 2011), mo-'nna (1 singulier + homme) devient ba-'nna (2 pluriel + homme) au pluriel, et kau (garçon) reçoit deux marqueurs préfixés différents li- et $m a-$ pour l'expression du nombre, lì-kau (5 singulier + garçon) et mà-kau (6 pluriel + garçon). Les marqueurs de classe sont des constituants d'un système d'accord qui se manifeste au niveau du syntagme nominal ou à celui de la phrase et sont partiellement liés au sémantisme du substantif. Ainsi, en tswana, des "noms de personne " sont attestés avec des marqueurs des classes 1 (mò-) (le pluriel est alors accompagné du marqueur de classe $2(b \grave{a}-))$, 5 (lì-) (avec le pluriel en 6 (mà-)), 7 (sì-) (avec le pluriel en 8-10 (dì/ dìn)) et 9 ( $n$ ou zéro) (avec pluriel en 8-10). Selon Creissels (1999: 182), « il y a de toute évidence une relation privilégiée entre les classes $1 / 2$ et la notion de personne humaine (ne serait-ce que du fait que tous les noms propres de personnes sans exception font leur accord en classes $1 / 2) »$.

Voici comment fonctionne le marqueur de classe $k i$ (classe 7) en kikongo (exemple 1) et en Swahili (exemple 2) dans le cadre des accords au niveau de la phrase :

\begin{tabular}{|l|l|l|l|l|l|l|}
\hline 1. & & & & & & \\
\hline & kìnkutu & kyámè & kímósi & kyà & mpémbè & kisòtòkèlè \\
\hline
\end{tabular}




\begin{tabular}{|l|l|l|l|l|l|l|}
\hline & 7-chemise & 7-ma & 7-une & 7-de & blanche & $1^{\text {ère }}$ personne-temps-tomber-aspect \\
\hline \\
'Une de mes chemises blanches est tombée.' \\
\hline
\end{tabular}

\begin{tabular}{|l|l|l|l|l|}
\hline 2. & \multicolumn{4}{|l|}{ Swahili (Welmers 1973: 171 cité par Van de Velde 2019) } \\
\hline & Ki-kapu & $k i$-moja & $k i$-kubwa & $k i$-li-anguk-a \\
\hline & 7-basket.NP & 7-big.NP & 7-one.SP & 7-PST-fall-FV \\
\hline \multicolumn{4}{|l}{} \\
\hline
\end{tabular}

Un certain nombre de langues bantoues rajoute un augment à la forme « préfixe + base substantivale »

\begin{tabular}{|l|l|l|l|l|}
\hline 3. & \multicolumn{4}{|l|}{ Bemba (Givón $1974: 132$, Kasonde 2009: 167 cité par Van de Velde 2019) } \\
\hline & a & ba-ntu & $b a$-suma & \\
\hline & AUG & 2-person.NP & 2-good & \\
\hline & \multicolumn{2}{|l}{} \\
\hline
\end{tabular}

\begin{tabular}{|l|l|l|l|l|}
\hline 4. & \multicolumn{4}{|l|}{ Bemba (Givón $1974: 132$, Kasonde $2009: 167$ cité par Van de Velde 2019) } \\
\hline & a & ba-ntu & a & $b a$-suma \\
\hline & AUG & 2-person.NP & AUG.NP & 2-good \\
\hline & \multicolumn{2}{|l}{ 'The people, who were (all) good.'; 'The people, the good ones.' } \\
\hline
\end{tabular}

41 Parfois, le marqueur de classe est post-posé à la base substantivale dans certaines langues bantoues.

\subsection{L'acquisition des marqueurs de classe dans les langues bantoues}

42 Les recherches sur l'acquisition des langues bantoues comme L1 et comme L2 contribuent à éclairer le fonctionnement des marqueurs de classe dans les langues bantoues. Dans une étude consacrée à l'acquisition des marqueurs en Sesotho, en Setswana et dans quelques autres langues bantoues par des enfants, K. Demuth (2000) montre que tous les apprenants de langues bantoues L1 omettent les marqueurs de classe, les marqueurs nominaux comme ceux qui expriment les accords de classe sur les autres unités lexicales, dans un premier temps de leur appropriation. Ensuite les entités substantivales reçoivent un marquage réduit avant d'être produits conformément à la norme. La morphonologie des classes nominales est manifestement 
acquise avant les éventuelles spécialisations sémantiques. Cela confirme la faible spécialisation sémantique des marqueurs de classe dans les langues bantoues. Tsonope (1993), qui est en accord avec la séquence développementale identifiée par Demuth, explique l'ordre d'acquisition relevé par des considérations accentuels et prosodiques.

Selon Spinner (2010), les apprenants de langues bantoues L2 omettent rarement l'élément préfixal du substantif mais plutôt les accords de classe. Une des explications de ce phénomène serait que les apprenants considèrent que le marqueur de classe fait partie de l'entité substantivale. Spinner rappelle également que les marqueurs de classe à valeur de singulier sont davantage utilisés que ceux à valeur de pluriel. Le comportement le plus fréquent des apprenants serait de sur-généraliser le marqueur de classe singulier hors de toute considération sémantique, y compris dans des contextes de pluralité, ce qui en fait un marqueur de pluriel par défaut erroné. Spinner relève enfin, que certains apprenants manifestent une certaine sensibilité au trait sémantique " animé » tel qu'il est marqué dans certaines langues bantoues et qu'ils ont tendance à généraliser les marqueurs de classe porteurs de ce sémantisme.

\subsection{Une convergence et une congruence entre le système nominal bantou et celui du français?}

Un examen, même sommaire, du système des marqueurs de classes des langues bantoues, permet de voir que la morphologie nominale de ces langues est différente de celle $\mathrm{du}$ français. Les classes nominales des langues bantoues et du français et leurs accords sont organisés différemment sur les plans morphologique et sémantique. Certes, on peut constater que les locuteurs de langues bantoues sont sensibles à des modifications préfixales du nom et ajoutent des augments à la base substantivale dans certains contextes et dans certaines langues. Cette propension cognitive est susceptible de se manifester lors de l'appropriation d'une langue étrangère. L'exemple de l'emprunt du lexème arabe kitab (livre) en Swahili permet de bien appréhender le fonctionnement des marqueurs de classe dans les langues bantoues. En effet, le lexème arabe est interprété comme un nom Swahili; il reçoit donc les marqueurs suivants: kitabu ( 7 singulier + livre) vs vitabu ( 8 pluriel + livre) sur la base d'une réanalyse phonique et sémantique de l'initial du mot arabe.

Sur la base de cette comparaison grammaticale et acquisitionnelle, il est permis d'interroger l'intuition de Baker (1984) à propos d'une éventuelle proximité entre les marqueurs de classe des langues bantoues et les déterminants du français qui se manifesterait par l'affixation nominale des lexèmes français lors de l'émergence des langues créoles IdFC. Certes, les locuteurs de langues bantoues ont tendance à porter leur perception sur l'initial des lexèmes mais il semble difficile de soutenir qu'il existe une convergence isomorphique, telle qu'elle a été définie supra, entre le $\mathrm{SN}$ des langues bantoues et le SN du français. On peut tout au plus considérer qu'il existe un air de famille entre les marqueurs de classe des langues bantoues et les déterminants du français. Par ailleurs, comme le montre entre autres, Mather (2005), en français L1 et L2, des faits d'agglutination sont attestés sans que l'on doive arguer de l'influence des langues bantoues. Il cite notamment des exemples empruntés à Lafage (1985), qui montrent que des locuteurs de l'éwé agglutinent l'article et le nom du français, en français langue étrangère (l'école, l'auto, nenfants) (Mather 2005 : 71). 


\section{L'agglutination nominale des créoles français : un phénomène multi-déterminé}

Remettre en question l'éventuelle congruence ou convergence entre la détermination nominale des langues bantoues et du français et le rôle de ces processus dans l'émergence de la classe nominale des créoles français conduit à penser que l'agglutination nominale des créoles français pourrait résulter d'une surdétermination où concourent de nombreux procédés, de la conservation de traits des langues mises en contact à la réanalyse.

\subsection{La classe nominale dans les créoles français}

Les créoles français, comme d'autre langues créoles, présentent une transcatégorialité telle qu'un même item lexical peut relever de plusieurs classes grammaticales (Véronique 2020). Ainsi en 5 et 5', le même item agglutiné fixe lager peut être un nom et un verbe,

$$
\begin{array}{|l|}
\text { 5. mau. en lager in leve (une bagarre a éclaté) } \\
\hline \text { 5'. mau. li n lager ek bug la (il s'est battu avec l'homme) }
\end{array}
$$

La classe des noms dans les créoles français n'est pas organisée par un système de genre et par des phénomènes d'accord, comme dans la langue donatrice de son lexique, le français (même si des traces d'opposition de genre subsistent en créole réunionnais). En dépit des affirmations de Baker $(1984,1994)$, selon laquelle les SN des créoles IdFC manifestent une influence bantoue, par l'ampleur de l'agglutination nominale qui s'y manifeste et l'emploi de ti- (petit) à l'instar d'un marqueur de classe (voir le Tableau 2 infra),

Tableau 2

Dérivés nominaux à l'aide de $t i-$

\begin{tabular}{|l|l|}
\hline sat 'chat' & ti-sat 'châton' \\
\hline piti 'enfant' & ti-piti 'enfant' \\
\hline fey 'feuille' & ti-fey 'marie-jeanne' \\
\hline ros 'pierre' & ti-ros 'caillou' \\
\hline
\end{tabular}

la classe nominale des langues créoles françaises n'est pas non plus organisée selon un système des classes nominales bantoues (cf. Grinevald 1999, Creissels 1999). Au sein des lexèmes nominaux agglutinés alternants des langues créoles, l'alternance entre la forme nue et la forme augmentée semble être contrainte selon les créoles par des facteurs phonologiques, syntaxiques et sémantiques. 


\subsection{L'agglutination nominale et la variation dans les créoles français}

Tous les créoles français connaissent des formes lexicales agglutinées alternantes et non alternantes, comme le montrent par exemple les relevés du tableau 3 pour les créoles des Petites Antilles.

Tableau 3

Variation de l'agglutination dans les Petites Antilles

(d'après l'Atlas linguistique des Petites Antilles et Bernabé 1987)

\begin{tabular}{|l|l|l|}
\hline français & Créole de Guadeloupe & Créole de Martinique \\
\hline plage & lapla3 / pla3 & lapla3 / pla3 \\
\hline rosée & laruze / lawuze / laroze & roze / wuze / laroze / lawuze \\
\hline foudre & lafud / fud & lafud / fud \\
\hline maison & kaj & kaj / lakaj \\
\hline place & laplas / plas & plas \\
\hline route & larut / rut / wut & larut / rut / wut \\
\hline vie & lavi / vi & lavi \\
\hline mort & mò & lanmò \\
\hline porte & pot & lapot \\
\hline
\end{tabular}

51 L'évaluation exacte de la différence numérique entre les formes substantivales agglutinées, fixes et alternantes des créoles IdFC et des autres créoles français est selon tout vraisemblance difficile à établir. Les relevés fournis par Baker (1984) sont essentiellement une approximation indicative. Ainsi, René (2018), par exemple dénombre un nombre de formes lexicales non alternantes supérieur à celui avancé par Baker (1984) pour le créole haïtien.

52 Selon Chaudenson (2007) et Albers (2019), en créole de la Réunion, les items lexicaux monosyllabiques sont susceptibles de connaitre l'agglutination pour des raisons de syllabation comme en 6 ,

6. pasyans i géri lagal (la patience guèrit la gale).

53 En réunionnais, comme dans d'autres créoles français, les noms de masse, les noms abstraits et les noms bien individués sont susceptibles d'apparaître sous une forme longue, c'est-à-dire agglutinée (Albers 2019). 
Pour les créoles des Petites Antilles, Bernabé (1987) (exemples 7, 7' et 8, 8') et ZribiHertz et Jean-Louis (2013) (exemples 9 et $9^{\prime}$ ) fournissent des exemples d'alternance de formes nues et agglutinées porteuses de différenciations sémantiques et syntaxiques (liées aux compabilités avec des déterminants défini et indéfini),

\begin{tabular}{|l|}
\hline 7. lajistis ké pini yo (la justice les punira) \\
\hline 7'. pa ni jistis an péyi-tala (il n'y a pas de justice dans ce pays) \\
\hline 8. latè séta tout moun (la terre est à tout le monde) \\
\hline 8'. yo achté on gran tè pou konsui lèkol-la (On a acheté un grand terrain pour construire l'école). \\
\hline 9. ni an nouvo plaj adan vil ta-a (il y a une nouvelle plage dans cette ville) (valeur sortale de plaj) \\
\hline 9'. Mari ka alé laplaj (Marie va à la plage) (valeur individualisée de laplaj) \\
\hline
\end{tabular}

55 Ces distinctions s'appliquent également au mauricien, mais avec moins de régularité selon Henri et Bonami (2019: 12). Selon ces auteurs, les contraintes qui conditionnent l'emploi des formes nues et augmentées des nominaux agglutinées pourraient trouver leur origine dans la valeur des déterminants français où une même unité exprime tantôt une valeur de défini pragmatique et tantôt une valeur de défini sémantique, opacité levée dans les créoles français par l'alternance des formes agglutinées et nues.

\subsection{Discussion}

56 L'analyse que propose Baker (1984) de l'agglutination nominale repose sur l'idée d'un transfert par les esclaves des structures nominales bantoues dans leur traitement de l'input français et sur l'existence d'une hypothétique convergence isomorphique entre le français et les langues bantoues. Cette séduisante hypothèse acquisitionnelle apparaît fragile à l'analyse. De plus, il est nécessaire de conserver à l'esprit que les français 'périphériques' susceptibles d'avoir constitué l'input fourni aux populations serviles alloglottes connaissent également des cas d'agglutination, hors de toute influence des langues bantoues. Neumann-Holzschuh et Mitko (2018), qui donnent cette analyse pour $z$-animaux, $z$-oreilles, lamer, $n$-oncle, lail, larb(r) etc. dans les français d'Acadie et de Louisiane, ajoutent : « ce qui est certain, c'est que l'agglutination est achevée dans les cas où l'ancienne consonne de liaison ou l'article élidé apparaissent dans tous les contextes et qu'ils n'alternent plus avec d'autres consonnes » (Neumann-Holzschuh \& Mitko 2018 : 15).

$57 \mathrm{Au}$ vu des faits examinés dans la section 5 à propos des classes nominales des langues bantoues, et des faits inventoriés supra, il semble raisonnable de considérer que l'agglutination nominale des créoles français, provient de multiples déterminations, de la conservation et de la généralisation intra-systémique d'usages du français, à la nécessité de marquer des différences sémantiques telles que le défini sémantique et le défini pragmatique, et à d'éventuels transferts partiels entre les langues des populations d'esclaves et le français colonial lors de la créolisation. 


\section{Conclusion}

Sur la base des recherches sur l'acquisition des langues étrangères, la convergence et la congruence apparaissent comme des mécanismes tardifs de la créolisation qui interviennent dans la phase où la forme grammaticale de la langue récipiendaire est déjà en place et que des transferts peuvent s'effectuer entre les langues sources et le créole émergeant. Pour Myers-Scotton (2002), la créolisation est entièrement due à la convergence. À la base de ce mécanisme, se trouverait la possibilité d'une influence translinguistique des langues en contact. La convergence de Myers-Scotton (2002), la congruence de Baptista (2020) et l'hybridation d'Aboh (2015) se présentent comme des mécanismes unitaires ; je pencherai pour ma part pour une vision de la convergence ou de la congruence dans la créolisation comme résultant de l'action de plusieurs mécanismes qui concourent tous à l'émergence de structures nouvelles. Étudier la créolisation à la lumière des recherches sur l'appropriation des LE par des adultes plurilingues suppose de bien appréhender les conditions qui président à la mobilisation de la convergence ou congruence et d'autres mécanismes linguistiques et cognitifs de transfert, d'analogie, de réanalyse, de convergence et de congruence. Des facteurs sociohistoriques sont également à prendre en compte dans la créolisation car ils façonnent la nature même des contacts inter-linguistiques.

\section{BIBLIOGRAPHY}

Aboh, E.O. (2015) The Emergence of Hybrid Grammars: Contact, Change and Creation, Cambridge: Cambridge University Press.

Aboh, E.O. \& M. DeGraff (2017) A null theory of creole formation based on Universal Grammar, in Roberts, I. (ed.) The Oxford handbook of universal grammar, p. 401-458, Oxford: Oxford University Press.

Albers, U. (2019) Le syntagme nominal en créole réunionnais : forme et interprétation, Thèse de doctorat, Aix-Marseille Université.

Andersen, R.W. (1983) Transfer to somewhere, in Gass, S. \& L. Selinker (eds) Language Transfer in Language Learning, p. 177-201, Rowley (MA): Newbury House.

Baissac, C. (1880) Étude sur le patois créole mauricien, Nancy: Berger-Levrault.

Baker, P. (1984) Agglutinated French articles in Creole French: Their evolutionary significance, Te Reo, 27, p. 89-129.

Baker, P. (1994) Creativity in creole genesis, in Adone, D. \& I. Plag (eds) Creolization and Language Change, p. 65-84, Tübingen: Niemeyer.

Baptista, M. (2020) Competition, selection, and the role of congruence in Creole genesis and development, Language, 96(1), p. 160-199. 
Baptista, M., Gelman, S.A. \& E. Beck (2016) Testing the role of convergence in language acquisition with implications for creole genesis, International Journal of Bilingualism, 20, 3, p. 269-296.

Becker, A. \& T. Veenstra (2003) The survival of inflectional morphology in French-related creoles, Studies in Second Language Acquisition, 25, p. 283-306.

Bernabé, J. (1987) Grammaire créole. Fondas kreyol la, Paris: L'Harmattan.

Bickerton, D. (1981) Roots of language, Ann Arbor: Karoma Press.

Bickerton, D. (1988) Creole languages and the bioprogram, in Newmeyer, F.J. (ed.) Linguistics: The Cambridge Survey. II Linguistic Theory: Extensions and Implications, p. 268-284, Cambridge: Cambridge University Press.

Bollée, A. (2007) Die Rolle der Konvergenz bei der Kreolisierung, in Ureland, Per Sture (ed.) Die Leistung der Strataforschung und der Kreolistik. Typologische Aspekte der Sprachkontakte. Akten des 5. Symposions über Sprachkontakt in Europa, p. 391-405, Tübingen: Niemeyer.

Carpooran, A. (2011) Diksioner Morisien. Premie diksioner kreol monoleng, Maurice: Les Editions le Printemps,

Chaudenson, R. (1992) Des îles, des hommes, des langues : essai sur la créolisation linguistique et culturelle, Paris: Editions L'Harmattan.

Chaudenson, R. (2007) Bare nouns in Réunionnais Creole, in Baptista, M. \& J. Guéron (eds) Noun Phrases in Creole Languages. A multi-faceted approach, p. 226-242, Amsterdam: J. Benjamins.

Corne C. (1999) From French to Creole. The development of new vernaculars in the French colonial world, London: Westminster University Press.

Creissels, D. (1991) Description des langues négro-africaines et théorie syntaxique, Grenoble: ELLUG.

Creissels, D. (1999) « Genres » indo-européens et « classes nominales » Niger-Congo, Faits de langues, 14 , p. 177-184

Creissels, D. (2011) Le Tswana, in Bonvini, E., Busuttil, J. \& A. Peyraube (eds) Dictionnaire des langues, p. 176-184, Paris: PUF.

Demuth, K. (2000) Bantu noun class systems: Loan word and acquisition evidence of semantic productivity, in Senft, G. (ed.) Systems of nominal classification, p. 270-292, Cambridge: Cambridge University Press.

Dubois, J., Giacomo, M., Guespin, L., Marcellesi, C., Marcellesi, J.B \& J.P. Mével (1973) Dictionnaire de Linguistique, Paris: Larousse.

Fournier, R. \& H. Wittmann (1982) L'agglutination nomale en français colonial (populaire et créole), Revue québécoise de linguistique théorique et appliquée, 2,2, 185-209.

Grinevald, C. (1999) Typologie des systèmes de classification nominale, Faits de langues, 14, p. 101-122

Gumperz, J.J \& R. Wilson (1971) Convergence and Creolization. A case from the Indo-Aryan/ Dravidian Border in India, in Hymes, D. (ed.) Pidginization and Creolization of languages. Proceedings of a conference held at the University of the West Indies, Mona, Jamaica, April 1968, p. 151-167, Cambridge: Cambridge University Press.

Haspelmath, M. (1998) Does grammaticalization need reanalysis?, Studies in Language, 22, 2, p. 315-351. 
Haugen, E. (1956) Bilingualism in The Americas: A Bibliography and Research Guide, Alabama: University of Alabama.

Kuteva, T. \& B. Heine (2007) The Genesis of Grammar: A Reconstruction, Oxford: Oxford University Press.

Henri, F. \& O. Bonami (2019) Prédire l'agglutination de l'article en mauricien, Faits de langues, 49, 1, p. 113-138.

Hopper, P.J \& E. Closs-Traugott (1993) Grammaticalization, Cambridge: Cambridge University Press. Jackendoff, R. (1999) Possible stages in the evolution of the language capacity, Trends in Cognitive Sciences, 3, 7, p. 272-279.

Jarvis, S. \& A. Pavlenko (2008) Crosslinguistic Influence in Language and Cognition. London, Routledge.

Johanson, L. (1998) Code-copying in Irano-Turkic, Language Science, 20, 3, p. 325-337.

Johanson, L. (2002) Do languages die of 'structuritis'? On the role of code-copying in language endangerment, Rivista di Linguistica, 14, 2, p. 249-270

Klein, W. \& C. Perdue (1997) The Basic Variety (or Couldn't natural languages be much simpler?), Second Language Research, 13, 4, p. 301-347.

Klein W. \& C. Perdue (1992) Utterance structure. Developing grammars again, Amsterdam: John Benjamins.

Kriegel, S., Ludwig, R. \& S. Pfander (2019) Dialectes, créolisation, convergences. Quelques hypotheses à partir du berrichon et du poitevin-saintongeais, in Dufter, A., Grübl, K., \& T. Scharinger (eds) Des parlers d'oil à la francophonie : Contact, variation et changement linguistiques, $\mathrm{p}$. 299-348, Berlin: De Gruyter.

Ladhams, J. (2012) Article agglutination and the African contribution to the Portuguese-based Creoles, in Bartens, A. \& P. Baker (eds) Black through White. African words and calques which survived slavery in Creoles and transplanted European languages, p. 31-50, London. Colombo: Battlebridge.

Lafage, S. (1985) Français écrit et parlé en pays éwé (Sud-Togo), Paris: SELAF

Lang, J. (2009) Les langues des autres dans la créolisation. Théorie et exemplification par le créole d'empreinte wolof à l'île Santiago du Cap Vert, Tübingen: Gunter Narr Verlag.

Le Dû, J. \& G. Brun-Trigaud (2011) Atlas linguistique des Petites Antilles, Editions du CHTS.

LePage, R. \& A. Tabouret-Keller (1985) Acts of Identity: Creole-Based Approaches to Language and Ethnicity, Cambridge: Cambridge University Press.

Mather, P.-A. (2005) Noun Phrases in L2 French and Haitian: Clues on the origin of Plantation Creol, Journal of Universal Language, 6, p. 53-64.

Matras, Y. (2009) Language Contact, Cambridge: Cambridge University Press.

Matthews, P.H. (2005) The Concise Oxford Dictionary of Linguistics, Oxford: Oxford University Press.

Muysken, P. (2000) Bilingual Speech. A typology of Code-mixing, Cambridge: Cambridge University Press.

Myers-Scotton, C. (2002) Contact linguistic. Bilingual encounters and grammatical outcomes, Oxford: Oxford University Press. 
Myers-Scotton, C. \& J. Jake (2009) A universal model of code-switching and bilingual language processing and production, in Bullock, B.E \& J.T. Almeida (eds) The Cambridge Handbook of Linguistic Code-switching, 336-357, Cambridge: Cambridge University Press.

Neumann-Holzschuh, I. \& J. Mitko (2018) Grammaire comparée des français d'Acadie et de Louisiane, Berlin: De Gruyter.

Odlin, T. (1989) Language transfer: Cross-linguistic influence in language learning, Cambridge: Cambridge University Press.

Olko, J., Borges, R. \& J. Sullivan (2018) Convergence as the driving force of typological change in Nahuatl, STUF, 71, 3, p. 467-507.

Philippson, G. (2011) Le Swahili, in Bonvini, E., Busuttil, J. \& A. Peyraube (eds) Dictionnaire des langues, p. 160-167, Paris: PUF.

Pienemann, M. (1998) Language processing and second language acquisition: Processability Theory, Amsterdam: John Benjamins.

René, J.F. (2018) L'agglutination en créole haïtien. Interface syntaxe / sémantique, Mémoire, Faculté de Linguistique Appliquée, Université d'état d'Haïti.

Schwartz, B.D. \& R. Sprouse (1996) L2 cognitive states and the full transfer/full access model, Second Language Research, 12, p. 40-72.

Sebba, M. (2009) On the notions of congruence and convergence in code-switching, in Bullock, B. \& A.J. Toribio (eds) The Cambridge Handbook of linguistic Code-switching, 40-57, Cambridge: Cambridge University Press.

Silva-Corvolán, S. (1994) Language Contact and Change: Spanish in Los Angeles, Oxford: Clarendon Press.

Spinner, P. (2010) Review article: Second language acquisition of Bantu languages: A (mostly) untapped research opportunity, Second Language Research, 27, 3, p. 418-430.

Tsonope, J. (1993) Children's acquisition of Bantu noun class prefixes, Botswana Notes \& Records, 25,1, p. 111-117.

Van de Velde, M. (2019) Nominal morphology and syntax, in Van de Velde, M., Bostoen, K., Nurse, D. \& G. Philippson (eds) The Bantu Languages, $2^{\text {e }}$ edition, p. 237-269, London: Routledge.

Véronique, G.D. (2007) Des racines du langage : la linguistique naturaliste de Derek Bickerton, in Histoire, Épistémologie, Langage, Tome XXIX, Fasc. 2, Le naturalisme linguistique et ses désordres, p. 163-176.

Véronique, G.D. (2013) Créolisation et créoles, in Simonin, J. \& S. Wharton (eds) Sociolinguistique du contact. Dictionnaire des termes et concepts, p. 143-178, Lyon: ENS Éditions.

Véronique, G.D. (2020) Transcatégorialité et développement des langues créoles : l'exemple du 'nombre' et des 'prépositions' pour et avec dans les créoles français, in Do-Hurinville, D.-T., Dao, H.-L. \& A. Rialland (eds) De la transcatégorialité dans langues. Description, Modélisation, Typologie, p. 195-210, Paris: Éditions de la Société de linguistique de Paris.

Weinreich, U. (1953) Languages in contact, New York: Linguistic Circle of New York.

Zribi-Hertz A. \& L. Jean-Louis (2013) From Noun to Name: definiteness marking in Modern Martinikè, in Cabredo Hofherr, P. \& A. Zribi-Hertz (eds) Crosslinguistic studies on Noun Phrase structure and reference, p. 269-315, Leiden: Brill. 


\section{NOTES}

1. Je remercie un.e évaluateur.e anonyme dont les remarques ont contribué à améliorer ce texte de façon significative. Les faiblesses qui persistent sont évidemment miennes.

2. Voici les dates de fondation et d'occupation des colonies françaises où sont apparues des langues créoles françaises: St Christophe / St Kitts (1620 / 1627), Dominique (1635-1763), Guadeloupe et Martinique (1635-), Guyane (1639-), Sainte Lucie (1650-1803), Louisiane (1672-1763), St Domingue / Haïti (1659-1804), Bourbon / Réunion (1665-), Ile de France / Maurice (1721-1814), Seychelles (1770-1814). Dans cette contribution, j'emploierai de façon synonyme «langues créoles» et "créoles». J'utiliserai également "créoles français », « langues créoles françaises ", « haïtien », « mauricien », « réunionnais » etc., pour désigner ces langues de façon individuelle ou générique.

3. Baissac $(1880: 2)$ qui signale ce phénomène dans le chapitre qu'il consacre à l'article en mauricien y voit une influence du malgache car il relève des faits analogues dans les emprunts du malgache au français.

4. Selon Henri et Bonami (2019: 2), « l'agglutination n'est pas à proprement parler un processus morphologique mais un phénomène diachronique de réinterprétation de frontières de mots qui a pour résultat la grammaticalisation de nouveaux mots sur la base d'une combinaison d'au moins deux mots ".

5. Les marqueurs de classe et les classes sont traditionnellement numérotés. "La numérotation des classes (...) est arbitraire, mais très répandue parmi les bantuistes; elle permet de faciles comparaisons d'une langue à l'autre ; ». (Philippson 2011 : 162).

\section{ABSTRACTS}

Congruence and convergence are frequently mentioned to explain crosslinguistic phenomena, such as code alternations, borrowings etc., which arise when languages are in contact (Matras 2009). As a rule, these linguistic processes are generated by the coexistence of languages in the same area over a long period of time (hence the creation of sprachbünde), and by situations of language acquisition and bilingualism. These processes are fueled by the acquisition strategies developed by plurilingual speakers (Haspelmath 1998). Congruence and convergence are often used as synonyms to name the linguistic factors that shape the material (linguistic matter) and the modes of organization (patterns) of languages in contact (Matras 2009: 148), but sometimes these terms may refer to distinct linguistic processes.

In this contribution, convergence and congruence are discussed in the light of the sociohistorical and linguistic development of Creole languages. The meaning and use of these notions are examined through a discussion of the case of nominal agglutination (i.e. the reanalysis of the French Determiner + Noun sequences into new lexical units), presented by Baker (1984) as an example of transfer of a Bantu substratum in the development of Isle de France Creole (i.e. French Creoles from Mauritius, Rodrigues and Seychelles).

Since Weinreich (1953) and Haugen (1956), language contacts are known to foster the emergence of diaphonemes and diamorphemes, that is linguistic units which span languages in contact and are perceived by bilingual speakers as identical. These interlingual phenomena bear both on linguistic materials and on modes of organization. The notion of conceptual change of Jarvis and Pavlenko (2008) describes the conditions that trigger these crosslinguistic effects. To pose that 
the appropriation of the dominant language of a colony by plurilingual subalterns is at the origin of creolization is to accept that the emergence of Creole languages follows a course where transfer, convergence and congruence between languages in contact, and other general learning processes are at play. The crystallization of these learner varieties is made possible because of the development of socialization among the slaves, and of the related sociolinguistic phenomenon of focussing (Lepage \& Tabouret-Keller 1985).

Gumperz and Wilson (1971) and Myers-Scotton (2002) define the socio-linguistic and cognitive dimensions of convergence while Baptista (2020) uses the notion of congruence to describe the same linguistic phenomena. For these researchers, convergence and congruence, are unitary processes that engender new combinations of units and functions in languages in contact. For other researchers (for instance, Olko, Borges and Sullivan 2018), convergence or congruence is the result of a sum of mechanisms of linguistic change, including grammaticalization, lexical and grammatical borrowing and reanalysis.

In the present paper, convergence and congruence are defined as processes based on targeted linguistic transfer which, according to the dynamics of the languages in contact, give rise to reanalysis of the model languages, leading to the creation of new grammatical meanings in the replica languages. If the link is direct between congruence, convergence and reanalysis, i.e. the reinterpretation of the units and categories of the model language, it is indirect vis a vis grammaticalization (Hopper \& Closs-Traugott 1993: 220). Convergence and congruence, like other processes, contribute to the development of grammaticalization in emerging languages.

Nominal agglutination in French-related Creoles is of two types: a) a consonant is added to a French etymon beginning with an initial vowel; b) a syllable derived from the French determiner (mau. di, li etc.) agglutinates with the French lexical etymon to form new Creole lexemes, examples lera (rat) (from) < (fr. le rat), dizef (eggs) < (fr. de l'œuf), lizje (eyes) < (fr. les yeux) etc. According to Baker (1984), the massive presence of agglutinated nominal forms of the second type in the Creoles of Mauritius, Rodrigues and the Seychelles is due to the presence of Bantuphone slaves in Mauritius during the period of formation of the so-called Isle de France Creole (IdFC), i.e. between 1773 and 1810. Baker (1984: 111) sketches an acquisition scenario whereby the Bantuphone slaves, the majority of the inhabitants of Ile de France at that time, reanalyze the Determiner + Noun sequences of French into unique lexical items because of the transfer of their knowledge of Bantu class markers, leading to a case of isomorphic convergence (Baptista 2020).

The paper attempts to show that factors other than convergence and congruence could be at play in the inception of nominal agglutination and more widely of creolization in French Creoles. In the light of the definitions of convergence and congruence proposed, the paper investigates the phenomenon of lexical reanalysis called nominal agglutination, keeping in mind that transfer presupposes the identification of similar sites in the target language and that any isomorphic convergence or any congruence implies that identities have been perceived between the languages in contact.

The paper claims that nominal agglutination in French Creoles cannot be considered to constitute a strict case of convergence or congruence in spite of Baker (1984), Myers-Scotton (2002) and Baptista (2020)'s analyses. A cursory examination of the system of class markers in Bantu languages shows that the nominal morphology of these languages is different from that of French. The nominal classes of the Bantu languages and the French NP and its agreement specifiers are organized differently on the morphological and semantic levels. of course, speakers of Bantu languages tend to be sensitive to prefixes in the model NP and tend to add augments to the substantival base of the model language in certain contexts. However, these arguments are not sufficient to prove that nominal agglutination in French-related Creoles a case of congruence or convergence. 
Nominal agglutination in French Creoles has been and is produced by multiple factors, from the conservation and the intra-systemic generalization of regional uses of French to possible partial transfers between the languages of the slave populations and colonial French during creolization. Understanding creolization as a case of acquisition of the dominant language of the colony by multilingual adults presupposes an understanding of the conditions that govern the mobilization of convergence or congruence and other linguistic and cognitive mechanisms of transfer, analogy, reanalysis, convergence and congruence. Sociohistorical factors are also to be taken into account in creolization because they shape the very nature of inter-linguistic contacts.

La convergence et la congruence sont enclenchées à l'occasion de contacts les langues parlées dans un territoire donné. Ces processus, qui trouvent leur origine dans les conduites d'appropriation des locuteurs plurilingues, peuvent être décrits soit de façon unitaire, soit comme associant différents mécanismes d'évolution linguistique. La convergence et la congruence sont interrogées dans cette contribution à partir du développement socio-historique et linguistique des langues créoles. L'exemple retenu pour illustrer la discussion est celui de l'agglutination nominale dans les créoles français. Il s'agit de comprendre la réorganisation des suites françaises Déterminant + Nom (mon père, du sel, la mort, du miel etc.) en unités lexicales nouvelles dans les langues créoles françaises. Ce fonctionnement morphologique a été attribuée à l'influence des langues bantoues parlées par les esclaves. L'article tente de montrer que d'autres facteurs que la convergence et la congruence pourraient être à l'origine de l'agglutination nominale dans les créoles français, et au-delà de la créolisation.

\section{INDEX}

Keywords: Creoles, creolisation, congruence, convergence, transfer, second language acquisition, grammaticalisation, nominal agglutination

Mots-clés: acquisition des langues secondes, créoles, créolisation, congruence, convergence, transfert, réanalyse, grammaticalisation, agglutination nominale

\section{AUTHOR}

\section{GEORGES DANIEL VÉRONIQUE}

Aix Marseille Univ, CNRS, LPL, Aix-en-Provence, France

georges.veronique@univ-amu.fr 